\title{
Effectiveness of Tai chi exercise on overall quality of life and its physical and psychological components among older adults: a systematic review and meta- analysis
}

\author{
Di Wang (ii) ${ }^{1}$, Pengcheng Wang (ii) ${ }^{2}$, Kun Lan (iD ${ }^{1}$, Yingchun Zhang (ii) ${ }^{1}$, and Yingli Pan (iD) ${ }^{1}$ \\ ${ }^{1}$ Fourth Affiliated Hospital, China Medical University, Shenyang, China \\ ${ }^{2}$ School of Nursing, Nanjing University of Chinese Medicine, Nanjing, China
}

\begin{abstract}
With the aging of the world's population, the quality of life of older adults is becoming more important. There are many studies on the use of Tai chi exercise, a popular form of mind-body exercise practiced by older adults. However, the effectiveness of Tai chi exercise on the quality of life of older adults is unclear. For this systematic review and meta-analysis, six databases (PubMed, CENTRAL, CINAHL, EMBASE, Scopus, CNKI) were searched in English and Chinese languages to screen for relevant randomized controlled trials (RCT), and their risk of bias was assessed by two independent reviewers. The results of quality of life, physical component, and psychological component among older adults were meta-analyzed using RevMan5.3 software. The search retrieved 2577 records. After screening, a total of 10 RCTs were included in this evaluation, with a total of 1170 participants. The meta-analysis showed that compared with the control group, Tai chi exercise had a significant impact on the overall quality of life $(S M D=1.23 ; 95 \% \mathrm{Cl}: 0.56-1.98 ; P<0.0001)$, and on the physical component of quality of life $(M D=5.90$; $95 \% \mathrm{Cl}: 1.05-10.75 ; \mathrm{P}=0.02)$, but no significant impact on the psychological component of quality of life. This study had high heterogeneity. The results of this study suggest the potential use of Tai chi exercise as an activity for increased quality of life in older adults. Future research may enhance experimental rigor and explore the rationale behind Tai chi exercise.
\end{abstract}

Key words: Tai chi exercise; Older adults; Quality of life; Physical component; Psychological component; Meta-analysis

\section{Introduction}

In recently years, the global population has been aging rapidly. One study showed that Pacific Asia is the most rapidly aging region globally with an estimated 200 million people over 65 years of age between 2015 and 2030 (1). Due to the normal aging process and a variety of chronic diseases, older adults usually face physical and psychological health challenges. Approximately 18 million adults over 65 years old have physical limitations (2). As reported, $52 \%$ have problems in walking, grasping, handing, or pushing, and $75.3 \%$ had balance impairment (3). Physical limitations and balance disorders can lead to a decline in functional capacity of older adults, impairing their independence.

Health maintenance and physical independence of older adults have become a widely discussed topic. According to the literature, the quality of life of the elderly can be influenced by a number of factors, among which various studies emphasize the role of demographic, physical, psychological, social, and religious characteristics (4). Quality of life (QoL) is an important component in the health status of older adults.

QoL can be divided into general QoL or health-related QoL (HRQoL). The former is based on a wide range of definitions, covering well-being and happiness without reference to health problems and disorders. HRQoL is part of a multidimensional approach that takes into account physical, psychological, and social factors as well as disease-related limitations. Clinicians are paying more and more attention to exercise, which has been identified as a safe and effective way to improve aerobic capacity, strength, and HRQoL $(5,6)$. According to the World Health Organization, participating in sports activities may play a key role in healthy aging to promote good QoL (7).

Tai chi is a traditional Chinese exercise and mind-body meditative exercise based on thousands of years of ancient history. A large number of studies have confirmed

Correspondence: Yingli Pan: <panyingli@sina.com>

Received April 19, 2020 | Accepted July 20, 2020 
that Tai chi exercise has a positive effect on chronic diseases in older adults (8). Based on interventional studies, several systematic reviews have been performed to produce high level evidence of the effectiveness of Tai chi exercise, in terms of immunity and infections (9), cardiovascular conditions (10), metabolic syndrome (11), bone mineral density in postmenopausal women (12), sleep disorders (8), certain cancers (13), etc. Further, a systematic review and meta-analysis by $\mathrm{Yu}$ et al. (14) found positive effects of Tai chi exercise in psychological well-being, such as reducing stress, anxiety, depression, and mood disturbance, and increasing self-esteem. Specifically, systematic reviews and meta-analyses have been published concerning the older adult population, with conclusions that Tai chi exercise may have positive effectiveness in improving their balance function and reducing falls (15), increasing their balance confidence, and in lowering resting blood pressure.

Concerning Tai chi exercise for QoL, several randomized controlled trials have been performed, of which conclusions are inconsistent with each other to some extent (16-18). On the other hand, previous reviews show that Tai chi exercise may play some positive role in improving life status. To our knowledge, however, the limitation of such reviews is that they have not specifically focused on Tai chi exercise as in intervention for QoL, or just considered sleep quality as one of the secondary outcomes, which means low clinical significance (8). No systematic review has solely investigated Tai chi exercise as the main intervention for QoL as primary outcome in any group of population, including older adults. Therefore, we performed this systematic review and meta-analysis to explore the effectiveness of Tai chi exercise to improve QoL in older adults.

\section{Material and Methods}

\section{Selection strategy}

With no time limit, we performed a comprehensive search of the medical literature in 5 English databases: PubMed, Cochrane Central Register of Controlled Trials (CENTRAL), Cumulative Index of Nursing and Allied Health Literature (CINAHL), Excerpta Medica Database (EMBASE), and Scopus, which have been checked from their inception up to December, 2019. We used the following MeSH (medical subject heading) terms and text words for searching: ("Tai Ji" or "Taijji" or "Tai Ji exercise" or "Taijiquan" or "Tai Ji Quan" or "Taichi" or "Tai chi" or "Tai Chi Chuan" or "shadow boxing") AND ("HRQoL" or "health related quality of life" or "quality of life" or "QoL" or "life quality" or "living quality"). We also searched the Chinese database "China National Knowledge Infrastructure (CNKI)". The CNKI database was searched to ensure a more robust review because Tai chi exercise is primarily rooted in Chinese culture so many articles are published only in Chinese databases. Subject heading terms and text words included: (“太极” (Taichi) OR “太极拳” (Taichi Chuan)) AND (“生活质量” (QoL) OR “与健康相关的生活质量” ( $\mathrm{HRQ}$ RL)). Finally, a snowball search was done.

\section{Eligibility criteria}

We included randomized controlled trials (RCTs) published from January to December, 2019, which met the following inclusion criteria: 1) studies conducted on participants who were aged 50 years and above and suffering from one or more chronic diseases or not; 2) studies evaluating Tai chi exercise interventions that were facilitated individually and/or in groups, guided by Tai chi exercise professional/non-professional, or in person and/ or using audio-visual material; 3) studies that compared Tai chi exercise with standard care, waitlist control, no intervention, newspaper reading, health education, routine activity, or walking; and 4) studies that reported at least one of the outcomes of QoL using validated measurement tools. Given that Tai chi exercise is of Chinese origin, studies published in English and Chinese were included to broaden the representativeness of evidence and prevent bias.

We excluded studies that utilized exercise intervention programs with or without Tai chi exercise as a component of mixed interventions (e.g., Tai chi exercise and acupuncture). Conference proceedings, abstracts only, book chapter reviews, letters, discussions, and editorials were also excluded.

\section{Study selection}

Search results were downloaded from the respective databases and imported into Endnote X9 software (Clarivate, USA). After removing duplicate articles, the titles and abstracts of studies were assessed independently by two authors (DW and PCW) for their relevancy to this review. Studies were then organized according to those that met, potentially met, or did not meet the eligibility criteria. The full-texts of all relevant and potentially relevant trials were retrieved and each of the two authors independently processed and analyzed the articles to determine if they were to be included in the review. A third author (YLP) was consulted for disagreements when a consensus was not established after discussion.

\section{Data collection process and data extraction}

A modified data collection form adapted from the Cochrane data extraction form published in the Cochrane Handbook (19) for Systematic Reviews of Interventions was used to guide extraction and collection of data from the individual trials. Data extraction was carried out independently by two reviewers (DW and PCW), to minimize bias and prevent errors. A third author (YLP) was consulted to resolve discrepancies. Study authors were contacted via emails (with a maximum of three attempts) in the event of missing information. To reduce extraction error and ensure 
relevancy of data included, the data extraction form was piloted by testing it on two included studies. Improvements were made to the data extraction form after piloting to tailor it better to the needs of this review.

\section{Quality assessment}

The Cochrane "Risk of Bias" assessment tool as denoted in the Cochrane Handbook of Systematic Reviews of Interventions was used to assess bias (19). The following domains were assessed; random sequence generation (selection bias), allocation concealment (selection bias), blinding of participants and personnel (performance bias), blinding of outcome assessment (detection bias), incomplete outcome data (attrition bias), and selective reporting (reporting bias).

Two reviewers (DW and PCW) independently assessed each study and made evidence-based judgements on the attempts of study authors to minimize bias in their trials. Any disagreements were discussed and resolved by a third author (YLP). Each study was methodically judged in all domains and placed into one of the following categories: low, high, or unclear risk of bias.

\section{Data synthesis}

We synthesized extracted data using meta-analytical methods. RevMan 5.3 software, provided by the Cochrane Collaboration (UK), was used for meta-analysis. Continuous data were analyzed using the inverse variance approach by combining the mean difference of individual studies when the outcome was reported using the same measurement scale, or the standardized mean difference (SMD) of individual studies, when the outcome was reported using different measurement scales. The SMD in the meta-analysis, also known as the Cohen's d, was used to evaluate the magnitude of effect size $(d<0.2$, very small effect size, $0.2 \leqslant d<0.5$, small effect size, $0.5 \leqslant d<0.8$, moderate effect size; $d \geqslant 0.8$, large effect size) (19).

Heterogeneity was evaluated through computation of the $\mathrm{I}^{2}$ statistic and chi-squared test, with consideration for effect magnitude and direction. A chi-squared test with a $\mathrm{P}$-value $<0.10$ (significance level of 0.1 ) indicated that a study was heterogeneous. The $\mathrm{I}^{2}$ statistic was used to assess the extent of heterogeneity $\left(\mathrm{I}^{2}=0-30 \%\right.$, low; $\mathrm{I}^{2}=30-60 \%$, moderate; $\mathrm{I}^{2}=50-90 \%$, substantial, $\mathrm{I}^{2}=75-$ $100 \%$, considerable). If heterogeneity was not significant (P-value $>0.10$ and $\mathrm{I}^{2}<30 \%$ ), the fixed-effects model was adopted. If heterogeneity was significant $(P$-value $<0.10$ and $\mathrm{I}^{2} \geqslant 30 \%$ ), a random-effects model was used.

\section{Results}

\section{Search process}

The systematic search yielded 2577 records: PubMed $(n=420)$, EMBASE $(n=879)$, CINAHL $(n=313)$, Cochrane $(n=670)$, Scopus $(n=112)$, and CNKI $(n=153)$. Thirty additional records were identified from other sources of reference lists. Duplicates (798) were removed, leaving 1779 records for screening. From those, 1000 records based on title and 644 records based on abstract were excluded after screening against the eligibility criteria. The full text of the remaining 135 records were retrieved, independently screened by two reviewers (DW and PCW), and 123 articles were included for the reasons as listed in Figure 1. Finally, 10 studies were eligible for inclusion in this review. The PRISMA Flowchart (Figure 1) illustrates the search process.

\section{Description of included studies}

The data from the 10 studies are summarized in Supplementary Table S1. All studies were individual RCTs; eight were two-arm RCTs (20-27), two were three-arm RCTs $(28,29)$. Three studies were conducted in China $(21,24,26)$, two in Hong Kong $(22,28)$, one in Korea (25), one in Germany (23), one in Spain (20), one in Iran (27), and one in the United States (29).

There were several types of Tai chi exercise in the 10 RCTs, including the combination of the 18 movements of Tai chi exercise (22), the 13 movements of Breathing Regulation Tai chi exercise (28), which were selected and modified from the 18 movements of Tai chi exercise produced by the Department of Health of Hong Kong, the 24 movements of Tai chi exercise $(21,23,26,27)$, and the 20 movements of Tai chi exercise, developed by Dr. Paul Lam (see ref. 25 for details). The comparable interventions in the control groups were usual care, exercise, slow walking, traditional physical exercise, and usual daily activities.

Tai chi exercise was studied in older adults, older adults with chronic obstructive pulmonary disease, older adults admitted to an intermediate care rehabilitation unit, older adults with congestive heart failure and depression, older adults with benign prostate hyperplasia, elderly females with low bone mass, highly maladjusted institutionalized elderly, and older adults with total knee arthroplasty. The length of the Tai chi exercise interventions ranged from 4 weeks to 24 weeks, with a median duration of 14.4 weeks for studies assessing HRQoL. The duration of the individual sessions ranged from 15 to 90 min, frequency from two to five times per week.

To evaluate HRQoL outcomes, the following instruments were used: Medical Outcomes 12-item Short Form Health Survey (SF-12), St. George's Respiratory Questionnaire Hong Kong Chinese (SGRQ-HKC), a visual analogical scale (VAS), Minnesota Living with Heart Failure Questionnaire (MLHF), Chinese version of WHOQOL-BREF, Urination-Related Quality of Life (QoL), Medical Outcomes 36-item Short Form Health Survey (SF-36), Activities of Daily Living (ADL), Neuropsychiatric Inventory(NPI), and Leiden Padua Quality of Life questionnaire (LEIPAD).

\section{Risk of bias}

Table 1 presents the risk of bias summary of the included studies. Eight studies detailed their random sequence 


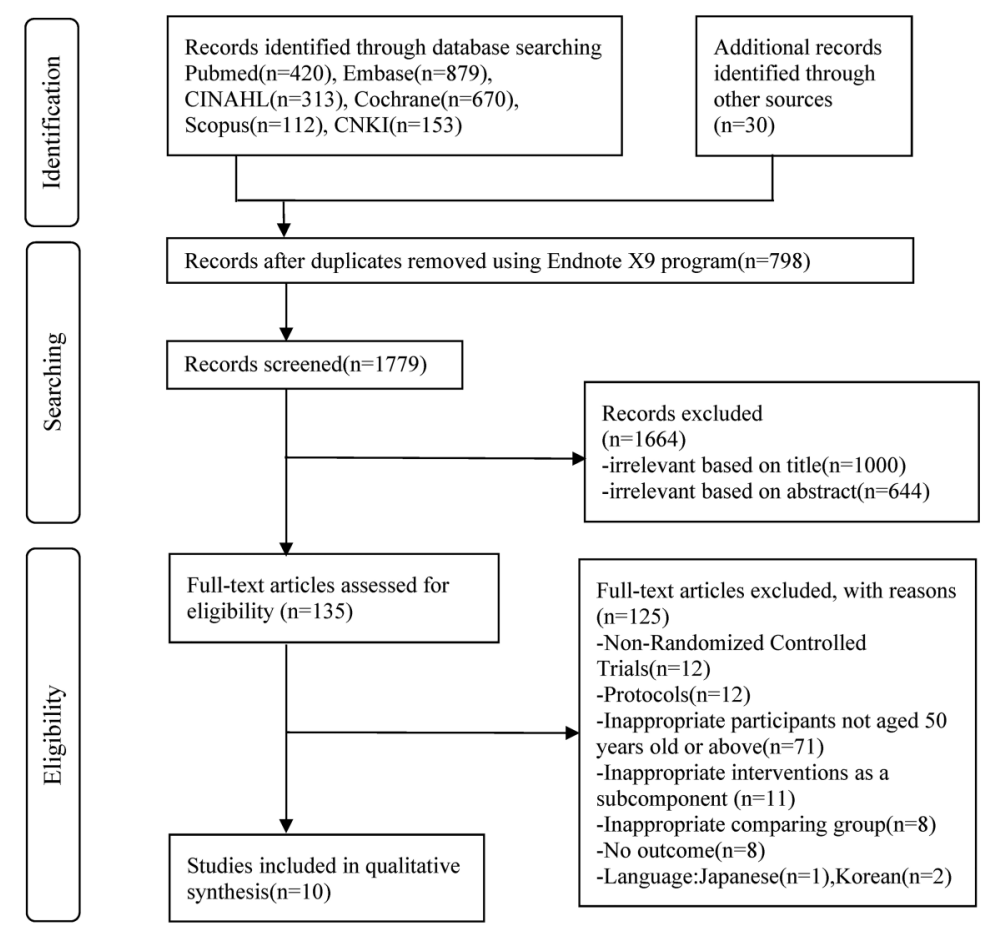

Figure 1. PRISMA flow diagram.

Table 1. Risk of bias assessment of included randomized clinical trials.

\begin{tabular}{|c|c|c|c|c|c|c|c|c|}
\hline & $\begin{array}{l}\text { Random } \\
\text { sequence } \\
\text { generation }\end{array}$ & $\begin{array}{c}\text { Allocation } \\
\text { concealment }\end{array}$ & $\begin{array}{l}\text { Blinding of } \\
\text { participants and } \\
\text { personnel }\end{array}$ & $\begin{array}{l}\text { Blinding of } \\
\text { outcome } \\
\text { assessment }\end{array}$ & $\begin{array}{c}\text { Incomplete } \\
\text { outcome data }\end{array}$ & $\begin{array}{l}\text { Selective } \\
\text { outcome } \\
\text { reporting }\end{array}$ & $\begin{array}{c}\text { Other } \\
\text { sources of } \\
\text { bias }\end{array}$ & Total \\
\hline Chan et al. 2017 (22) & + & + & - & + & + & + & + & 6 \\
\hline Chan et al. $2010(28)$ & + & + & + & $?$ & + & + & + & 6 \\
\hline Martinez et al. 2015 (20) & + & + & + & + & + & + & + & 7 \\
\hline Yuan et al. 2016 (21) & + & - & $?$ & - & + & + & + & 4 \\
\hline Cui et al. 2017 (24) & $?$ & - & - & $?$ & + & + & + & 3 \\
\hline Jung et al. 2012 (25) & + & - & - & + & + & + & + & 4 \\
\hline Chyu et al. 2010 (23) & + & + & $?$ & - & + & + & + & 5 \\
\hline Dechamps et al. 2010 (29) & $?$ & - & $?$ & $?$ & + & + & + & 3 \\
\hline Li et al. $2019(26)$ & + & + & $?$ & - & + & + & + & 5 \\
\hline Tajik et al. 2018 (27) & + & + & - & + & + & + & + & 6 \\
\hline
\end{tabular}

+ : Low risk of bias; ?: Unclear risk of bias; -: High risk of bias (mean score for risk of bias was 4.9).

generation process (20-23,25-28) and six studies described allocation concealment measures $(20,22,23$, 26-28). Due to the nature of Tai chi exercise intervention, it was difficult to blind participants, resulting in only two studies describing the blinding of participants and personnel, and the others obtaining either an unclear or high risk of performance bias $(20,28)$. Three studies reported blinding of outcome assessors $(22,25,27)$. All studies had a low incomplete outcome data bias, a low selective outcome reporting bias, and a low other sources of bias (20-29).

\section{Effectiveness of Tai chi exercise on overall QoL}

We performed the meta-analysis on overall QoL. Different scales were used in the six studies to measure and evaluate the QoL. In four of the studies $(20,24,27,28)$, the higher the score, the better the QoL, while in the other two studies $(21,25)$, the higher the score, the worse 


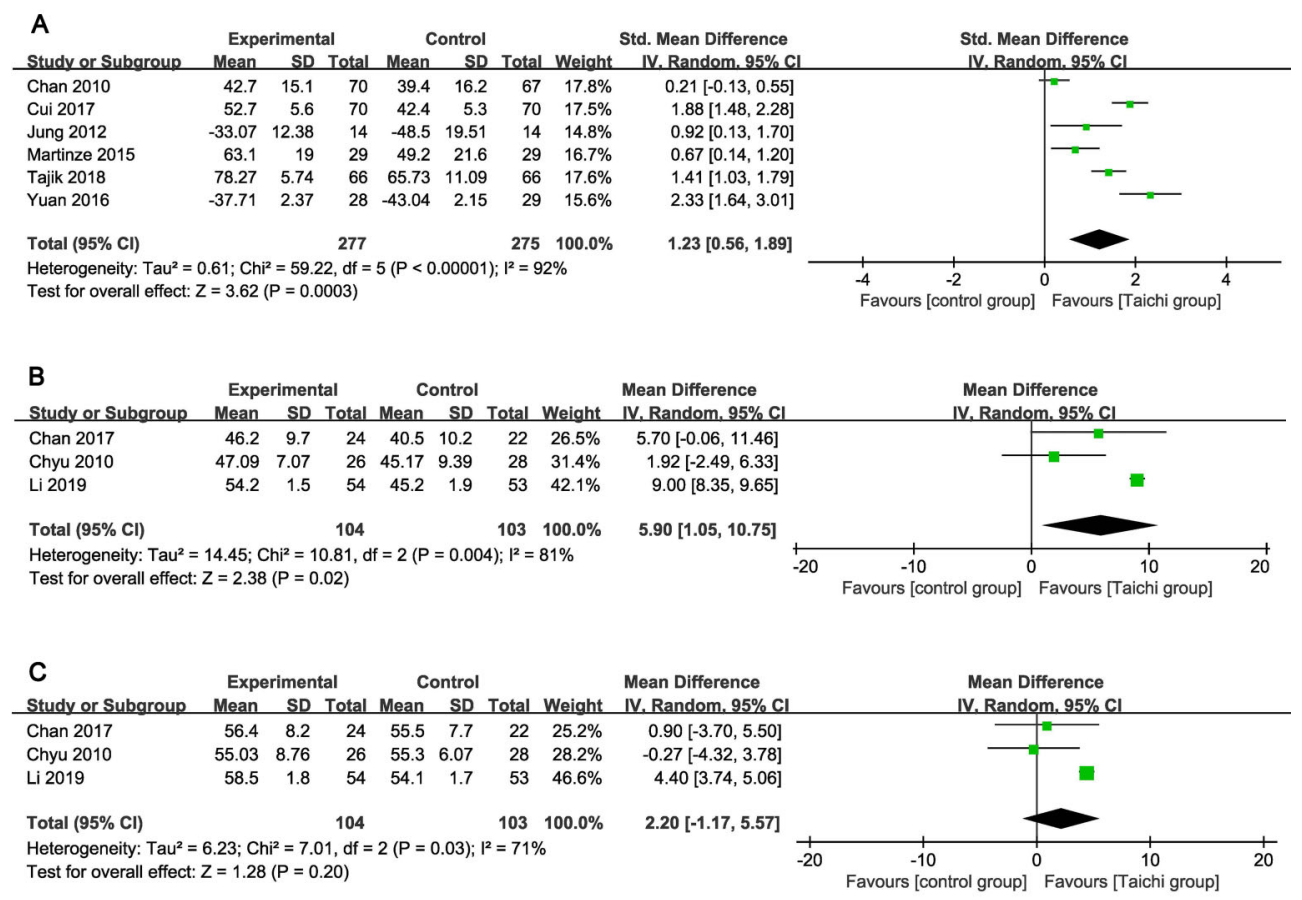

Figure 2. Forest plot of the effectiveness of Tai chi exercise on quality of life in older adults. See Table 1 for reference numbers.

the QoL. For the two studies $(21,25)$, we took the mean times as described in the Cochrane Handbook (16) and converted these values into the new value, with the standard deviation unchanged. Six studies involving 277 participants in the Tai chi exercise group and 275 participants in the control group were meta-analyzed for overall QoL. The pooled results showed statistically significant effect favoring Tai chi exercise (SMD=1.23; 95\%Cl: 0.56-1.98; $\mathrm{P}<0.0001)$. Significant and substantial heterogeneity was found among the combined studies $\left(I^{2}=92 \% ; P=0.0003\right)$ (Figure 2A).

\section{Effectiveness of Tai chi exercise on physical} component and psychological component of QoL

Three studies involving 104 participants in the Tai chi exercise group and 103 participants in the control group were meta-analyzed for the physical component and the psychological component $(22,23,26)$. The Tai chi exercise group showed better performance on the physical component $\left(\mathrm{MD}=5.90 ; 95 \% \mathrm{Cl}\right.$ : $1.05-10.75 ; \mathrm{I}^{2}=81 \%$; $\mathrm{P}=0.02$ ) compared with the control group (Figure 2B). When combining all three RCTs in pool analysis, the direction on the mental component favored the Tai chi exercise compared with the controls, but since the $95 \%$ confidence interval included zero, the difference was not significant $\left(\mathrm{MD}=2.20 ; 95 \% \mathrm{Cl}:-1.17-5.57 ; \mathrm{I}^{2}=71 \%\right)$ (Figure 2C).

\section{Sensitivity analysis}

Sensitivity analysis on overall QoL. The fixed effect model was used to recombine the statistics. The Tai chi exercise group was statistically significant in improving QoL (SMD=1.10; 95\% Cl: 0.91-1.28; $\mathrm{P}<0.01)$. This was the same as the results of the random effects model, indicating that the results were stable.

Sensitivity analysis on physical component. The fixed effect model was used to recombine the statistics. The Tai chi exercise group was statistically significant in the physical component $(\mathrm{MD}=8.81 ; 95 \% \mathrm{Cl}$ : 8.17-9.45; $\mathrm{P}<0.01)$. This was the same as the results of the random effects model, indicating that the results were stable.

\section{Discussion}

This review aimed to examine the effectiveness of Tai chi exercise on the QoL among adults aged 50 and above with or without chronic diseases. The 10 RTCs studies involved 1030 participants. The duration of interventions ranged from four weeks to twenty-four weeks with varying forms of Tai chi exercise.

Six studies that measured overall QoL using the correlation scales were meta-analyzed revealing a positive effect of Tai chi exercise $(20,21,24,25,27,28)$. Although the analysis showed high heterogeneity, the sensitivity analysis showed the same efficacy. All six studies used 
different tools to measure self-efficacy, which may have led to the high degree of heterogeneity. This result is consistent with a recent review on Tai chi exercise for women with cancer that also reported significant improvements in QoL with Tai chi (30). Chronic disease compromises QoL by slowly decreasing life control and the ability to participate in meaningful activities and pursue desired outcomes. All participants in the assessment were over 50 years old, which meant they may lack control in their lives. One plausible reason for the current findings is that participants who completed Tai chi exercise interventions regained personal sensory control, which is an important determinant of QoL in older adults, increasing their confidence in disease management and improving people's overall quality of life.

The meta-analysis of three studies showed a good effect in improving physical components, which are part of QoL. Significant heterogeneity was detected, which may be related to diverse forms and durations of Tai chi interventions and different health conditions among older adults.

Although the difference was not statistically significant, more than $50 \%$ of individual RCTs reported that Tai chi exercise improved psychological components related to QoL. Other similar reviews found that psychological components such as depression were significantly reduced in studies comparing Tai chi exercise to passive controls $(15,31)$. The possible reason for the difference in the significance of the results may be the differences in the studies included in the evaluation. Further high-quality studies are needed to develop conclusive evidence and reconcile the differences on Tai chi exercise in alleviating psychological components in older adults.

\section{Limitations}

There are several limitations to this systematic review. Most studies were conducted in Asia and the review was

\section{References}

1. Gouw VXH, Jiang Y, Seah B, He H, Hong J, Wang W. Effectiveness of internal Qigong on quality of life, depressive symptoms and self-efficacy among community-dwelling older adults with chronic disease: a systematic review and meta-analysis. Int J Nurs Stud 2019; 99: 103378, doi: 10.1016/j.jijnurstu.2019.06.009.

2. Hamer M, Bates CJ, Mishra GD. Depression, physical function, and risk of mortality: National Diet and Nutrition Survey in adults older than 65 years. Am J Geriatr Psychiatry 2011; 19: 72-78, doi: 10.1097/JGP.0b013e3181df465e.

3. Dillon $\mathrm{CF}, \mathrm{Gu} \mathrm{Q}$, Hoffman $\mathrm{HJ}$, Ko CW. Vision, hearing, balance, and sensory impairment in Americans aged 70 years and over: United States, 1999-2006. NCHS Data Brief 2010; 1-8; Available from: < https://pubmed.ncbi.nlm. nih.gov/20377973/>

4. Jing W, Willis R, Feng Z. Factors influencing quality of life of elderly people with dementia and care implications: a systematic review. Arch Gerontol Geriatr 2016; 66: 23-41, doi: 10.1016/j.archger.2016.04.009. limited to studies in English and Chinese languages. The limited number of RCTs and the heterogeneity in outcome measurements, Tai chi exercise interventions, intervention durations, frequency of weekly class, and methodological rigor made it difficult to determine the effectiveness of Tai chi exercise on QoL outcomes in older adults and to recommend minimum effective dosage, types, intensity, or format (sitting, standing). In addition, trials of complementary therapies published outside mainstream journals may be biased systematically.

\section{Implications for practice and research}

Tai chi exercise is a safe, economical, and beneficial exercise that is easy to learn. According to this systematic review, Tai chi exercise can be considered a sport to improve the QoL of older adults. However, before recommending Tai chi exercise, healthcare professionals should make different recommendations based on the situation and diagnosis of older adults. Future randomized controlled trials should provide more rigorous research procedures, in particular measures to reduce bias, so that more accurate assessments of quality and intervention effectiveness can be achieved.

\section{Conclusions}

This systematic review demonstrated that Tai chi may be an exercise option for older adults, with a positive effect on overall QoL and physical component. However, for the psychological component, the effect was not significant.

\section{Supplementary Material}

Click here to view [pdf].

5. Rogers LQ, Hopkins-Price $P$, Vicari $S$, Pamenter $R$, Courneya KS, Markwell $S$, et al. A randomized trial to increase physical activity in breast cancer survivors. Med Sci Sports Exerc 2009; 41: 935-946, doi: 10.1249/MSS.0b 013e31818e0e1b.

6. Speck RM, Gross CR, Hormes JM, Ahmed RL, Lytle LA, Hwang WT, et al. Changes in the Body Image and Relationship Scale following a one-year strength training trial for breast cancer survivors with or at risk for lymphedema. Breast Cancer Res Treat 2010; 121: 421-430, doi: 10.1007/s10549-009-0550-7.

7. Galloza J, Castillo B, Micheo W. Benefits of exercise in the older population. Phys Med Rehabil Clin N Am 2017; 28: 659-669, doi: 10.1016/j.pmr.2017.06.001.

8. Du S, Dong J, Zhang H, Jin S, Xu G, Liu Z, et al. Taichi exercise for self-rated sleep quality in older people: a systematic review and meta-analysis. Int $J$ Nurs Stud 2015; 52: 368-379, doi: 10.1016/j.ijnurstu.2014.05. 009. 
9. Ho RTH, Wang CW, Ng SM, Ho AHY, Ziea ETC, Wong VT, et al. The effect of tai chi exercise on immunity and infections: a systematic review of controlled trials. $J$ Altern Complement Med 2013; 19: 389-396, doi: 10.1089/acm. 2011.0593.

10. Liu T, Chan AW, Liu YH, Taylor-Piliae RE. Effects of Tai Chibased cardiac rehabilitation on aerobic endurance, psychosocial well-being, and cardiovascular risk reduction among patients with coronary heart disease: a systematic review and meta-analysis. Eur J Cardiovasc Nurs 2018; 17: 368383, doi: $10.1177 / 1474515117749592$.

11. Lauche R, Peng W, Ferguson C, Cramer H, Frawley J, Adams $\mathrm{J}$, et al. Efficacy of Tai Chi and Qigong for the prevention of stroke and stroke risk factors: a systematic review with meta-analysis. Medicine 2017; 96: e8517, doi: 10.1097/MD.0000000000008517.

12. Wayne PM, Kiel DP, Krebs DE, Davis RB, Savetsky-German $\mathrm{J}$, Connelly M, et al. The effects of Tai Chi on bone mineral density in postmenopausal women: a systematic review. Arch Phys Med Rehabil 2007; 88: 673-680, doi: 10.1016/ j.apmr.2007.02.012.

13. Wayne PM, Lee MS, Novakowski J, Osypiuk K, Ligibel J, Carlson LE, et al. Tai Chi and Qigong for cancer-related symptoms and quality of life: a systematic review and metaanalysis. J Cancer Surviv 2018; 12: 256-267, doi: 10.1007/ s11764-017-0665-5.

14. Yu X, Chau JPC, Huo L. The effectiveness of traditional Chinese medicine-based lifestyle interventions on biomedical, psychosocial, and behavioral outcomes in individuals with type 2 diabetes: a systematic review with metaanalysis. Int J Nurs Stud 2018; 80: 165-180, doi: 10.1016/ j.ijnurstu.2018.01.009.

15. Gogulla S, Lemke N, Hauer K. Effects of physical activity and physical training on the psychological status of older persons with and without cognitive impairment [in German]. Z Gerontol Geriatr 2012; 45: 279-289, doi: 10.1007/s00391012-0347-x.

16. Luo L, Zou L, Fang Q, Wang H, Liu Y, Tian Z, et al. Effect of Taichi softball on function-related outcomes in older adults: a randomized control trial. Evid Based Complement Alternat Med 2017; 2017: 4585424, doi: 10.1155/2017/4585424.

17. Lee EO, Chae YR, Song R, Eom A, Lam P, Heitkemper M. Feasibility and effects of a tai chi self-help education program for Korean gastric cancer survivors. Oncol Nurs Forum 2010; 37: E1-E6, doi: 10.1188/10.ONF.E1-E6.

18. Sun J, Buys N, Jayasinghe R. Effects of community-based meditative Tai Chi programme on improving quality of life, physical and mental health in chronic heart-failure participants. Aging Ment Health 2014; 18: 289-295, doi: 10.1080/13607 863.2013.875120.

19. Higgins JPT, Green S (Editors), Higgins. Cochrane handbook for systematic reviews of interventions, version 5.1.0. The Cochrane Collaboration; <https://www.science-open. com/document?vid=539e5df3-6827-41af-87e4-11ee81754c cb2013>.

20. Martinez N, Martorell C, Espinosa L, Marasigan V, Domenech S, Inzitari M. Impact of Qigong on quality of life, pain and depressive symptoms in older adults admitted to an intermediate care rehabilitation unit: a randomized controlled trial. Aging Clin Exp Res 2015; 27: 125-130, doi: 10.1007/ s40520-014-0250-y.
21. Yuan LH, Zhang HB, Zhou F, Xue XJ, et al. Effect of Tai Chi on improvement of depression, sleeping quality and quality of life in elderly patients with chronic congestive heart failure complicated with depression [in Chinese]. Guangxi Med J 2016; 38: 1547-1550, doi: 10.11675/j.issn.0253-4304.2016. 11.19.

22. Chan AW, Yu DS, Choi KC. Effects of tai chi qigong on psychosocial well-being among hidden elderly, using elderly neighborhood volunteer approach: a pilot randomized controlled trial. Clin Interv Aging 2017; 12: 85-96, doi: 10.2147/CIA.S124604.

23. Chyu MC, James CR, Sawyer SF, Brismee JM, Xu KT, Poklikuha G, et al. Effects of tai chi exercise on posturography, gait, physical function and quality of life in postmenopausal women with osteopaenia: a randomized clinical study. Clin Rehabil 2010; 24: 1080-1090, doi: 10.1177/ 0269215510375902.

24. Cui JH, Fu LM. Effect of Taijiquan and slow walking on Chinese elderly female's cardiovascular function and quality of life. Biomed Res 2017; 28: 9525-9528; Available from: $<$ https://www.biomedres.info/abstract/effect-of-taijiquanand-slow-walking-on-chinese-elderly-females-cardiovascular-function-and-quality-of-life-9298.html > .

25. Jung S, Lee EN, Lee SR, Kim MS, Lee MS. Tai chi for lower urinary tract symptoms and quality of life in elderly patients with benign prostate hypertrophy: a randomized controlled trial. Evid Based Complement Alternat Med 2012;2012: 624692, doi: 10.1155/2012/624692.

26. Li L, Cheng S, Wang G, Duan G, Zhang Y. Tai chi chuan exercises improve functional outcomes and quality of life in patients with primary total knee arthroplasty due to knee osteoarthritis. Complement Ther Clin Pract 2019; 35: 121125, doi: 10.1016/j.ctcp.2019.02.003.

27. Tajik A, Rejeh N, Heravi-Karimooi M, Samady Kia P, Tadrisi SD, Watts TE, et al. The effect of Tai Chi on quality of life in male older people: a randomized controlled clinical trial. Complement Ther Clin Pract 2018; 33: 191-196, doi: 10.1016/j.ctcp.2018.10.009.

28. Chan AW, Lee A, Suen LK, Tam WW. Effectiveness of a Tai chi Qigong program in promoting health-related quality of life and perceived social support in chronic obstructive pulmonary disease clients. Qual Life Res 2010; 19: 653664, doi: 10.1007/s11136-010-9632-6.

29. Dechamps A, Diolez P, Thiaudiere E, Tulon A, Onifade C, Vuong $T$, et al. Effects of exercise programs to prevent decline in health-related quality of life in highly deconditioned institutionalized elderly persons: a randomized controlled trial. Arch Intern Med 2010; 170: 162-169, doi: 10.1001/archinternmed.2009.489.

30. Thongteratham NPK, Olson K, Ratanawichitrasin A, Nityasuddhi D, Wattanakitkrilert D. Effectiveness of Tai Chi Qi Qong program for thai women with breast cancer: a randomized control trial. PRIJNR (Internet) 2015; 19: 280-294; < Available from: https://he02.tci-thaijo.org/index. php/PRIJNR/article/view/25888 > .

31. Song R, Grabowska W, Park M, Osypiuk K, Vergara-Diaz GP, Bonato P, et al. The impact of Tai Chi and Qigong mindbody exercises on motor and non-motor function and quality of life in Parkinson's disease: a systematic review and metaanalysis. Parkinsonism Relat Disord 2017; 41: 3-13, doi: 10.1016/j.parkreldis.2017.05.019. 